\title{
Erratum
}

\section{Clinical trials and drug promotion: Selective reporting of Study 329}

Jon N. Jureidini, Leemon B. McHenry and Peter R. Mansfield

[International Journal of Risk \& Safety in Medicine 20(1,2) (2008), 73-81]

When this article was originally published, the following statement was omitted:

Baum Hedlund paid L.B.M. and J.N.J. to analyse documents relating to Study 329, but there was no funding to discuss, prepare, or write this article, the idea for which originated with the authors. 